\title{
Vitamin D status and bone mineral density (BMD) in a sub-set of participants from the Trinity Ulster Department of Agriculture Study (TUDA)
}

\author{
E. Laird ${ }^{1}$, M. Ward ${ }^{1}$, H. McNulty ${ }^{1}$, M. Healy ${ }^{2}$, M. C. Casey $^{2}$, J. J. Strain ${ }^{1}$ and J. M. W. Wallace ${ }^{1}$ \\ ${ }^{1}$ Northern Ireland Centre for Food and Health (NICHE), University of Ulster, Coleraine BT52 1SA and ${ }^{2}$ The Mercers \\ Institute for Research on Ageing (MIRA) and the Department of Biochemistry, St James's Hospital, Dublin, Ireland
}

Osteoporosis and osteopenia are increasingly common conditions associated with decreased BMD and increased bone fragility. Bone health is influenced by genetic, environmental and nutritional factors most notably Ca and vitamin $\mathrm{D}^{(1)}$. Within Ireland and the UK synthesis of vitamin D is limited to 6 months of the year which impacts negatively on 25-hydroxyvitamin D (25(OH)D) status. Indeed previous studies have highlighted $25(\mathrm{OH}) \mathrm{D}$ deficiency and insufficiency within the Irish population ${ }^{(2)}$. The objective of this study was to assess $25(\mathrm{OH}) \mathrm{D}$ status in a sub-sample $(n$ 420) of participants aged $>60$ years from the Northern Ireland cohort of the TUDA study, a large observational study of older adults. Participants were recruited between March 2009 and June 2010. Serum 25(OH)D concentration was assessed by HPLC MS (API 4000, AB SCIEX, USA). BMD of the total hip, femoral neck and lumbar vertebrae was measured using total body dual-energy X-ray absorptiometry (DXA) scans (Lunar Prodigy, GE Healthcare, UK).

\begin{tabular}{lcccccc}
\hline & All $(n$ 420) & SD & Males $(n$ 199) & SD & Females $(n$ 221) & SD \\
\hline Age (years) & 68.2 & 5.3 & 68.1 & 0.3 & 68.2 & 0.3 \\
BMI $\left(\mathrm{kg} / \mathrm{m}^{2}\right)$ & 30.5 & 5.1 & 29.8 & 0.2 & $31.2 * *$ & 0.3 \\
Serum PTH $(\mathrm{pg} / \mathrm{ml})$ & 51.4 & 2.1 & 48.8 & 1.9 & 53.7 & 2 \\
Serum Ca $(\mathrm{mmol} / \mathrm{l})$ & 2.3 & 0.01 & 2.3 & 0.01 & 2.3 & $<0.01$ \\
Serum $25(\mathrm{OH}) \mathrm{D}(\mathrm{nmol} / \mathrm{l})$ & 45.2 & 2.1 & 48.9 & 1.3 & $41.9^{* *}$ & 1.4 \\
\hline Differences between sex determined by independent samples $t$ test: $* * P<0.01 * P<0.05$ & &
\end{tabular}

Serum 25(OH)D concentration was significantly higher in men than in women $(P<0.05)$. Overall $>63 \%$ of the total sample were classed insufficient $(<50 \mathrm{nmol} / \mathrm{l})$ in $25(\mathrm{OH}) \mathrm{D}$ status with $25 \%$ females $v .11 \%$ of males classified deficient $(<25 \mathrm{nmol} / \mathrm{l})$. Results from DXA scans found that $48 \%$ of the total sample were osteopenic; osteoporosis was more common in females than males $(20 \% v .9 \%)$. There was a significant association (Partial Pearson correlation correcting for age, sex, BMI and 25(OH)D)) between 25(OH)D concentration and BMD at two sites; the femoral neck $(r=0.132, P<0.01)$ and the total hip $(r=0.097, P<0.05)$. A multinomial logistic regression analysis, controlling for BMI, age, sex and supplement indicated that a higher $25(\mathrm{OH}) \mathrm{D}$ concentration was associated with decreased odds of osteopenia ( $\beta-0.015$; OR 0.986). In conclusion, within this sample of older adults where both deficiency and insufficiency of $25(\mathrm{OH}) \mathrm{D}$ were evident, vitamin D status was significantly associated with BMD. Given the importance of $25(\mathrm{OH}) \mathrm{D}$ status in the prevention of osteoporosis, the results highlight the importance of addressing $25(\mathrm{OH}) \mathrm{D}$ deficiency in the older population.

We would like to acknowledge the Irish Department of Agriculture and the Northern Ireland Department for Employment and Learning through its 'Strengthening the all-Island Research Base' initiative the co-funding for this research.

1. Laird E, Ward M, McSorley E et al. (2010) Nutrients 2, 693-724.

2. O’Sullivan M, Nic Suibhne T, Cox G et al. (2008) Ir J Med Sci 177, 131-134. 